\title{
Trends in measures of handgrip strength from 2014 to 2017 among Korean adolescents using the Korean National Health and Nutrition Examination Survey Data
}

\author{
Yunkoo Kang ${ }^{1,2}$, Jieun $\mathrm{Kim}^{3}$, Seung Kim², Sowon Park², Hyunjung Lim, ${ }^{3,4^{*}}$ (I) and Hong Koh ${ }^{2 *}$ (1)
}

\begin{abstract}
Object: Measuring handgrip strength is a useful method to evaluate sarcopenia. No study has shown the trends of handgrip strength to weight ratio among Korean adolescents by year. This study aimed to determine the trends of handgrip strength among Korean adolescents using data from the Korea National Health and Nutrition Examination Survey (KNHANES). Data of 2304 adolescents who participated in the KNHANES between 2014 and 2017 were obtained. The handgrip-to-weight ratios were categorized by age, sex, and year.
\end{abstract}

Results: Handgrip strength in adolescents decreased from $28.67 \mathrm{~kg}$ in 2014 to $27 \mathrm{~kg}$ in 2017 (P for trend <0.05). The handgrip-to-weight ratio also decreased from 51.48 in 2014 to 48.18 in 2017 (P for trend <0.05). The handgrip strength and handgrip-to-weight ratio also decreased among boys and girls over the years 2014-2017 (P for trend <0.05). The results of the present study indicate that the handgrip-to-weight ratio decreased in Korean adolescents from the years 2014 to 2017, and a declining overall ratio indicates a decrease in the health status among Korean adolescents. Hence, there is a need to review the health status of Korean adolescents.

Keywords: Sarcopenia, Adolescent, Muscles, Handgrip Strength

\section{Introduction}

Sarcopenia is defined as a progressive and generalized skeletal muscle disorder that involves the accelerated loss of muscle mass and function [1]. Furthermore, sarcopenia is known to be associated not only with aging but also with other systemic diseases, such as metabolic syndrome and non-alcoholic fatty liver disease (NAFLD) in adults $[2,3]$. Thus, evaluating sarcopenia is important, for which there are many measurement tools available.

\footnotetext{
*Correspondence: hjlim@khu.ac.kr; khong@yuhs.ac

2 Department of Pediatrics, Severance Children's Hospital, Yonsei

University College of Medicine, 50-1 Yonsei-ro, Seodaemun-gu, Seoul 03722, South Korea

${ }^{4}$ Department of Medical Nutrition, Graduate School of East-West Medical Science, Kyung Hee University, Yongin, Gyeonggi-do 17104, South Korea Full list of author information is available at the end of the article
}

Computed tomography, magnetic resonance imaging, and dual-energy X-ray absorptiometry can measure muscle mass. Additionally, handgrip strength and knee flexion/extension strength can be used to assess muscle strength $[4,5]$. Handgrip strength assessment is a very simple and reliable method because it is correlated with several medical conditions [6,7], even in adolescents and children [8-11]. Thus, handgrip strength has been used to identify sarcopenic obesity in children [12]. A recent study reported a strong relationship between handgrip strength and obesity and metabolic syndrome in adults and adolescents [13-17]. Steffl et al. showed that handgrip strength can help identify children at risk for sarcopenic obesity [12]. The handgrip strength represents the strength of the simple muscle force, however, it can also represent something more important. The decreases

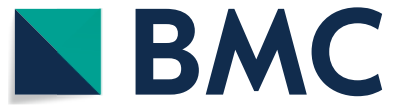

(c) The Author(s) 2020. This article is licensed under a Creative Commons Attribution 4.0 International License, which permits use, sharing, adaptation, distribution and reproduction in any medium or format, as long as you give appropriate credit to the original author(s) and the source, provide a link to the Creative Commons licence, and indicate if changes were made. The images or other third party material in this article are included in the article's Creative Commons licence, unless indicated otherwise in a credit line to the material. If material is not included in the article's Creative Commons licence and your intended use is not permitted by statutory regulation or exceeds the permitted use, you will need to obtain permission directly from the copyright holder. To view a copy of this licence, visit http://creativeco mmons.org/licenses/by/4.0/. The Creative Commons Public Domain Dedication waiver (http://creativecommons.org/publicdomain/ zero/1.0/) applies to the data made available in this article, unless otherwise stated in a credit line to the data. 
in grip strength can be attributed to problems with several medical conditions, such as children's mental health, obesity and metabolic syndrome as mentioned above. Based on this, if we can figure out the trends of handgrip strength, medical condition of children can be evaluated easily. However, no study has shown the trends of handgrip strength to weight ratio among Korean adolescents by year. This study aimed to evaluate the trends of handgrip strength to weight ratio and estimate the overall health condition among Korean adolescents.

\section{Main text Methods \\ Data}

The present study evaluated data from the 2014-2017 Korea National Health and Nutrition Examination Survey (KNHANES). Different participants were selected every year. These annual cross-sectional surveys are performed using multi-stage probability samples that are representative of the noninstitutionalized civilian Korean population. The data of selected participants has specific sampling weights factors because each data does not have equal probability of being selected. The data were analyzed after adjusting with specific sampling weights factors for each participant.

\section{Subject selection}

During 2014-2017, a total of 2988 individuals participated in the KNHANES. The present study included participants aged 10-18 years; participants with missing data were excluded. Thus, 2304 participants (1227 boys and 1077 girls) were included in the analysis. The participants were categorized by age and sex. The subjects were categorized into 9 age groups by each year, from ages 10 to 18 years.

\section{Measurement of anthropometric and laboratory variables}

Systolic blood pressure (SBP, $\mathrm{mmHg}$ ) and diastolic blood pressure $(\mathrm{DBP}, \mathrm{mmHg}$ ) were measured to the nearest $2 \mathrm{mmHg}$ using a mercury sphygmomanometer [Baumanometer Wall Unit 33(0850)]. Height was measured to the nearest $0.1 \mathrm{~cm}$ using a stadiometer (SECA 225, Seca $\mathrm{GmbH} \& \mathrm{KG}$.), and weight was measured to the nearest $0.1 \mathrm{~kg}$ using a balance beam scale (GL-600-20, G-tech). Waist circumference was measured to the nearest $0.1 \mathrm{~cm}$ using a measuring tape (SECA 200, Seca GmbH \& KG.). The body mass index (BMI) and waist-to-height ratio (WtHR) were calculated from the measured height and weight of participants $\left(\mathrm{kg} / \mathrm{m}^{2}\right.$ and waist $(\mathrm{cm}) /$ height $(\mathrm{cm}) \times 100$, respectively). Blood samples were collected from venous blood and levels of fasting glucose $(\mathrm{mg} /$ $\mathrm{dL}$ ), aspartate aminotransferase (AST, U/L), and alanine aminotransferase (ALT, U/L), triglyceride (TG, mg/dL), cholesterol (mg/dL), high-density lipoprotein (HDL, mg/ $\mathrm{dL}$ ), were measured using a Hitachi Automatic Analyzer 7600 [18].

\section{Handgrip strength measurement}

The Takei digital grip strength dynamometer (Model T.K.K.5401, Takei Co., Ltd., Ishioka, Japan) was used to measure handgrip strength. The dynamometers were calibrated according to a standardized protocol and a special investigator checked the measurement to ensure it was done properly. Measurement of handgrip strength was performed after rest and light exercise. All participants, except those with a history of wrist surgery within 3 months or any wrist discomfort, underwent the handgrip strength test. The handgrip strength testing procedures was done according to the muscle strength procedures manual by Centers for Disease Control and Prevention [19, 20]. Handgrip strength was measured in a standing position with the arm and wrist in the anatomical position. Participants were asked to exhale and apply a maximal grip for $3 \mathrm{~s}$, for a total of 3 repetitions each, starting with the dominant hand. The left and right hands were alternated. Sixty seconds of rest was allowed between each measurement. The highest handgrip strength value (in $\mathrm{kg}$ ) between both hands was recorded and included in the analysis [12] (http:// knhanes.cdc.go.kr/). Handgrip strength was calculated as a ratio (handgrip-to-weight, HGtW, (hand grip, HG/ weight) $\times 100)$ which was used in the analysis $[12,15]$.

\section{Statistical analysis}

The SPSS software (version 23.0; IBM Inc., Armonk, NY) was used for statistical analyses of all data. Participants have different sampling probabilities of being selected, therefore each participant have proper weight factor. In order to represent the entire Korean adolescent population with small number of participants, data was adjusted with sampling weight factors. One-way analysis of variance was used to compare the mean value of the continuous variables of SBP, DBP, height, weight, waist, BMI, WHtR, glucose, cholesterol, TG AST, ALT. Continuous data was analyzed and expressed as mean \pm standard error, applying weight factors. P for trend were calculated among years by linear regression with applying weight factors of survey design.

\section{Results}

\section{Characteristics of the participants from 2014 to 2017}

The characteristics of the study participants from 2014 to 2017 are shown in Table 1. All values including age, SBP, DBP, height, weight, waist, BMI, glucose, TG, AST, ALT did not show any trends over the years 2014, 2015, 2016, and 2017. However, WtHR decreased and total 
Table 1 Participants characteristics in KNHANES 2014 to 2017

\begin{tabular}{lrrrrrrr}
\hline & Total $(\mathbf{n}=\mathbf{2 3 0 4})$ & $\mathbf{2 0 1 4}(\mathbf{n}=\mathbf{4 8 3})$ & $\mathbf{2 0 1 5}(\mathbf{n}=\mathbf{5 7 4})$ & $\mathbf{2 0 1 6}(\mathbf{n}=\mathbf{6 3 4})$ & $\mathbf{2 0 1 7}(\mathbf{n}=\mathbf{6 1 3})$ & $\boldsymbol{B}$ coefficient & $\boldsymbol{P}$ for trend \\
\hline Age (year) & $14.41 \pm 0.06$ & $14.28 \pm 0.14$ & $14.41 \pm 0.13$ & $14.47 \pm 0.10$ & $14.49 \pm 0.14$ & 0.067 & 0.272 \\
SBP (mmHg) & $108.54 \pm 0.26$ & $108.06 \pm 0.61$ & $108.90 \pm 0.49$ & $109.14 \pm 0.53$ & $108.05 \pm 0.49$ & 0.008 & 0.975 \\
DBP (mmHg) & $66.41 \pm 0.22$ & $65.70 \pm 0.50$ & $66.31 \pm 0.40$ & $66.94 \pm 0.41$ & $66.67 \pm 0.47$ & 0.349 & 0.098 \\
Height (cm) & $162.12 \pm 0.28$ & $161.69 \pm 0.64$ & $161.80 \pm 0.53$ & $162.32 \pm 0.50$ & $162.66 \pm 0.50$ & 0.343 & 0.180 \\
Weight (kg) & $56.08 \pm 0.36$ & $55.63 \pm 0.86$ & $57.14 \pm 0.67$ & $55.58 \pm 0.68$ & $55.96 \pm 0.65$ & -0.069 & 0.838 \\
Waist (cm) & $71.05 \pm 0.26$ & $70.47 \pm 0.55$ & $72.73 \pm 0.49$ & $70.91 \pm 0.49$ & $70.09 \pm 0.51$ & -0.319 & 0.188 \\
BMI (z-score) & $0.12 \pm 0.32$ & $0.10 \pm 0.06$ & $0.30 \pm 0.68$ & $0.01 \pm 0.06$ & $0.05 \pm 0.06$ & 0.117 & 0.115 \\
WtHR & $43.83 \pm 0.14$ & $43.55 \pm 0.28$ & $44.97 \pm 0.28$ & $43.69 \pm 0.27$ & $43.10 \pm 0.28$ & -0.280 & 0.028 \\
Glucose (mg/dL) & $91.85 \pm 0.23$ & $92.33 \pm 0.67$ & $91.88 \pm 0.34$ & $91.82 \pm 0.36$ & $91.37 \pm 0.41$ & -0.293 & 0.218 \\
Cholesterol (mg/dL) & $162.27 \pm 0.66$ & $157.52 \pm 1.37$ & $161.59 \pm 1.23$ & $164.01 \pm 1.31$ & $165.95 \pm 1.35$ & 2.755 & $<0.001$ \\
TG (mg/dL) & $86.08 \pm 1.29$ & $85.52 \pm 2.95$ & $88.29 \pm 2.69$ & $84.89 \pm 2.26$ & $85.61 \pm 2.41$ & -0.337 & 0.777 \\
AST (U/L) & $19.36 \pm 0.23$ & $18.87 \pm 0.39$ & $19.82 \pm 0.66$ & $19.14 \pm 0.34$ & $19.60 \pm 0.40$ & 0.143 & 0.438 \\
ALT (U/L) & $15.73 \pm 0.46$ & $14.87 \pm 0.74$ & $16.36 \pm 1.22$ & $15.55 \pm 0.70$ & $16.12 \pm 0.94$ & 0.284 & 0.465 \\
\hline
\end{tabular}

Data presented as mean \pm standard error with weighting of survey design

SBP systolic blood pressure, DBP diastolic blood pressure, $B M I$ body mass index, WtHR waist to height ratio $($ waist $(\mathrm{cm}) / \mathrm{height}(\mathrm{cm}) \times 100), T G$ triglyceride, $A S T$ aspartate aminotransferase, $A L T$ alanine aminotransferase

cholesterol increased significantly between the years 2014 and 2017 (P for trend $<0.05$ ) (Table 1).
Trends of handgrip strength among boys from 2014 to 2017 The trends in HG and HGtW among the boys are shown by year and age (Fig. 1a, b). The overall (age 10 to 18) trends of boy's HG and HGtW ratio is decreasing over 2014 to 2017. HG and HGtW ratio decreased from

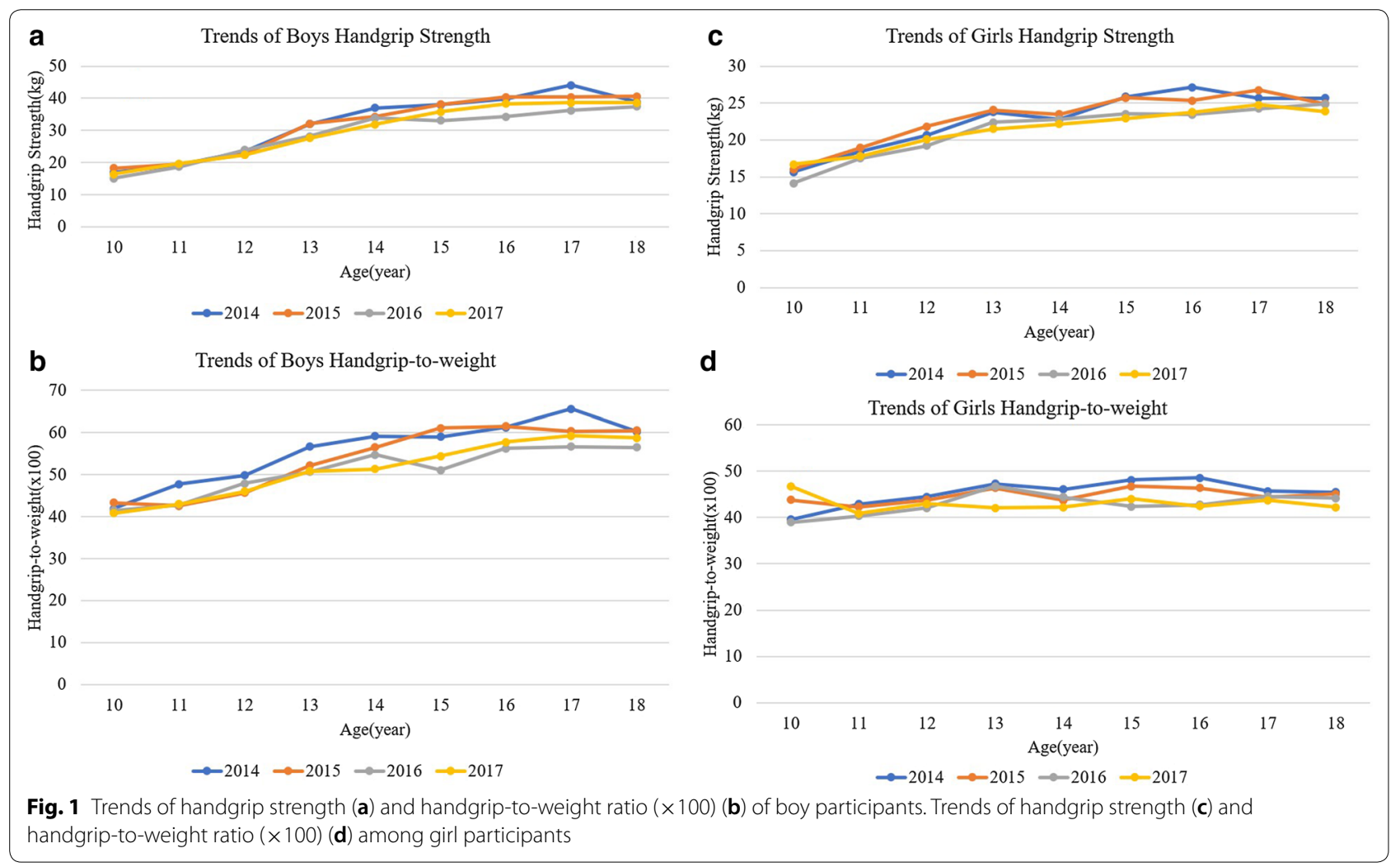


$33.3 \mathrm{~kg}$ to $31.6 \mathrm{~kg}$ and from 56.5 to 52.8 , respectively (P for trend $<0.05$ ) (Fig. 2a). The HG significantly decreased among 13-, 14-, 15-, and 17-year-old boys. Furthermore, HGtW ratio decreased in 13-, 14-, 15-, 16-, and 17-yearold boys ( $\mathrm{P}$ for trend $<0.05$ ) (Additional file 1: Tables S1, S2).

\section{Trends of handgrip strength among girls from 2014 to 2017}

The trends of HG and HGtW ratio among the girls are shown by year and age (Fig. 1c, d). The results showed a similar trend for HG and HGtW ratio among the girls as with the boys over the years ( $\mathrm{P}$ for trend $<0.05$ ). The HG decreased from $23.38 \mathrm{~kg}$ to $21.92 \mathrm{~kg}$ and HGtW ratio decreased from 45.7 to 43.1 (Fig. 2b). The HG decreased significantly in 13-, 14-, 15-, 16-, and 17-year-old girls. Furthermore, HGtW ratio decreased in 13-, and 16-yearold girls (P for trend $<0.05$ ) (Additional file 1: Tables S3, S4).

\section{Trends of handgrip strength among adolescents from 2014 to 2017}

The trends of HG and HGtW ratio among the overall adolescents (boys and girls) decreased over the years significantly, from $28.7 \mathrm{~kg}$ to $27.0 \mathrm{~kg}$ and from 51.5 to 48.2, respectively ( $\mathrm{P}$ for trend $<0.05$ ) (Fig. 2c) (Additional file 1: Table S5).

\section{Discussion}

Anthropometric measurements, such as height and weight are basic but important examinations for children and adolescents. Measuring height and weight may help in screening several diseases such as nonalcoholic fatty liver disease (NAFLD), metabolic syndrome. These non-invasive methods of obtaining information can be a good evaluation method for patients as well as pediatricians, especially for children and adolescents in whom we need to minimize invasive screening. However, even though various study shows importance of HG, it is still not used much compared to height, body weight, BMI, SBP, DBP. Some studies have demonstrated a relationship between muscle mass and current health status $[2,15]$. Cohen et al. showed the importance of muscle strength being associated with metabolic risk factors in children [21]. Moreover, Grontved et al. showed that adolescents' muscle strength is associated with cardiovascular risk in young adulthood [22]. In addition to the
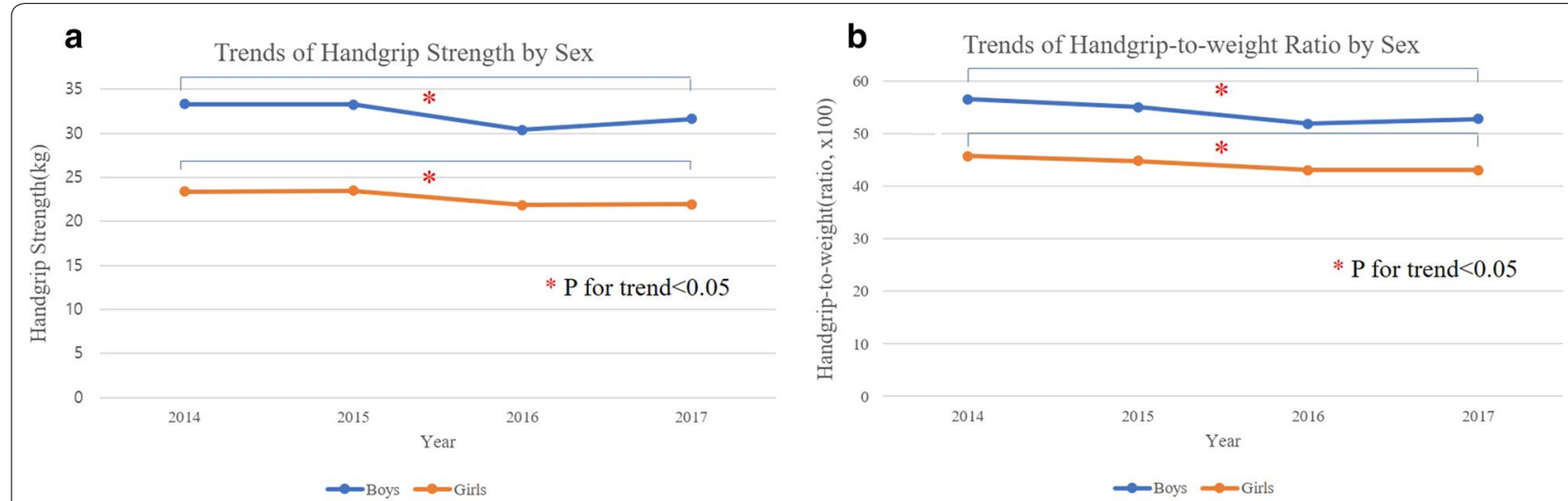

C

50

40

30

20

10

0

Trends of Handgrip Strength

2016

$\rightarrow$ Handgrip Strength $(\mathrm{kg})$

$\rightarrow$ Handgrip-to-weight(ratio, x100)

2017

* P for trend $<0.05$

Fig. 2 Trends of handgrip strength $(\mathbf{a})$ and handgrip-to-weight ratio $(\times 100)(\mathbf{b})$ by sex. Trends of handgrip strength and handgrip-to-weight ratio among adolescents (boys and girls) over the years 2014 to 2017 (c). The HG and HGtW significantly decreased from 2014 to 2017 (*P for trend<0.05) P for trend was analyzed by linear regression with applying weight factors of survey design 
above-mentioned metabolic syndrome-related diseases, many studies have demonstrated HG's relationship with functional and psychological health as well as quality of life [12, 13, 17, 23]. This study showed a significant decrease in HG and HGtW ratio among adolescent participants over the years from 2014 to 2017. The decrease in HG and HGtW indicates muscle strength has been decreased. Moreover, it might indicate that some of the disorders mentioned above are increasing.

A recent study of handgrip cutoffs performed in children and adolescents in Colombia reported that the lower the HGtW ratio, the higher the likelihood of cardiometabolic risk [24]. Compared with the HG cut-off presented in a previous study, the HGtW ratio among boys and girls aged $10-12$ years are higher ( 0.376 for boys and 0.359 for girls). In the present study, from 2014 to 2017, boys over 13 years of age showed an HGtW higher than the cut-off value ( 0.447 for boys) in a previous study. Girls' HGtW, however, was higher than the cut-off value ( 0.440 for girls) in year 2014, but the HGtW was found to be low in 2017. HGtW of Korean adolescents are higher than the cut-off value seen in the Colombia study, but it is declining over the years. Specifically, in 2017, HGtW of girls over 13 years of age were lower than the cut-off value.

Other variables except WtHR and cholesterol did not show significant trends over years, but HG and HGtW are decreasing over years and it can be affected by various variables as mentioned above. Therefore, attention is required even though there has been no significant change in blood pressure and laboratory test results in Korean adolescents. Currently, the situation in Korea does not seem to be irrelevant. The health status can be easily inferred by HG and HGtW, and this study implies the risk of health problems of Korean adolescents have begun to develop.

To summarize, the results of the present study showed a decrease in HG and HGtW in Korean adolescents, which might be indicative of a problem in overall health status among Korean adolescents. Decrease in HG and HGtW might be related to decreased physical activity levels or poorer dietary intakes. To our knowledge, this is first study to evaluate trends of HG and HGtW in adolescents and to show changes over the years. The overall decrease in HG and HGtW suggests deterioration of the health status among Korean adolescents. Hence, there is a need to review the health of Korean adolescents, and measures should be taken to prevent its deterioration.

\section{Limitations}

This study has a few limitations. First, in the KNHANES, muscle mass was not measured using dual-energy X-ray absorptiometry or imaging studies.
Second, there is a lack of information on the cut-off value of $\mathrm{HGtW}$ in Korean adolescents. If additional data are collected or cohorts are built in the future, it may be possible to determine cut-off values. Third, missing data were present that could affect the result. Further effort is needed to minimize the missing data because it represents the whole Korean adolescent. Fourth, puberty can affect the result of handgrip strength, however, tanner staging data is missing in KNHANES data. Fifth, physical activity, dietary intake or socioeconomic status can affect handgrip strength, but these variables were excluded in analysis [25]. However, despite these limitations, this is the first large-scale study to evaluate trends of handgrip strength in Korean adolescents.

\section{Supplementary information}

Supplementary information accompanies this paper at https://doi. org/10.1186/s13104-020-05112-3.

Additional file 1: Table S1. Handgrip strength in boys. Table S2. Handgrip strength to weight in boys. Table S3. Handgrip strength in girls. Table S4. Handgrip strength to weight in girls. Table S5. Handgrip strength and handgrip strength to weight in adolescents.

\section{Abbreviations}

KNHANES: Korean adolescents using data from the Korea National Health and Nutrition Examination Survey; NAFLD: Nonalcoholic fatty liver disease; SBP: Systolic blood pressure; DBP: Diastolic blood pressure; BMI: Body mass index; WtHR: Waist-to-height ratio; AST: Aspartate aminotransferase; ALT: Alanine aminotransferase; TG: Triglyceride; HDL: High-density lipoprotein; HG: Handgrip strength; HGtW: Handgrip-to-weight.

\section{Acknowledgements}

Not applicable.

\section{Authors' contributions}

$\mathrm{KH}$ and $\mathrm{LH}$ were the group leader. Conceptualization was done by KJ, KY, LH and KH. Formal analysis performed by KY. KJ, KS and PS worked with software. All authors approved the final manuscript as submitted and agree to be accountable for all aspects of the work. All authors read and approved the final manuscript.

\section{Funding}

Not applicable.

Availability of data and materials

The KNHANES data used in this study is available in http://knhanes.cdc.go.kr.

\section{Ethics approval and consent to participate}

The study was approved by the Yonsei Severance Hospital Institutional Review Board (No. 4-2018-1143). KNHANES surveys are national open-access public data approved by the Korean Center for Disease Control and informed consent was obtained by participants and parents or legal representative of children under 14 which is approved by Korean law. The raw data from the KNHANES surveys are freely available at http://knhanes.cdc.go.kr/.

\section{Consent for publication} Not applicable.

Competing interests

The authors declare no competing interest associated with this report. 


\begin{abstract}
Author details
${ }^{1}$ Department of Pediatrics, Yonsei University Wonju College of Medicine, Wonju, South Korea. ${ }^{2}$ Department of Pediatrics, Severance Children's Hospital, Yonsei University College of Medicine, 50-1 Yonsei-ro, Seodaemun-gu, Seoul 03722, South Korea. ${ }^{3}$ Research Institute of Medical Nutrition, Kyung Hee University, Seoul 02447, South Korea. ${ }^{4}$ Department of Medical Nutrition, Graduate School of East-West Medical Science, Kyung Hee University, Yongin, Gyeonggi-do 17104, South Korea.
\end{abstract}

Received: 9 March 2020 Accepted: 26 May 2020

Published online: 30 June 2020

\section{References}

1. Cruz-Jentoft AJ, Sayer AA. Sarcopenia. Lancet. 2019;393(10191):2636-46.

2. Lee YH, Kim SU, Song K, Park JY, Kim DY, Ahn SH, Lee BW, Kang ES, Cha BS, Han KH. Sarcopenia is associated with significant liver fibrosis independently of obesity and insulin resistance in nonalcoholic fatty liver disease: nationwide surveys (KNHANES 2008-2011). Hepatology. 2016;63(3):776-86.

3. Han E, Lee YH, Kim G, Kim SR, Lee BW, Kang ES, Ahn CW, Cha BS. Sarcopenia is associated with albuminuria independently of hypertension and diabetes: KNHANES 2008-2011. Metabolism. 2016:65(10):1531-40.

4. Cruz-Jentoft AJ, Baeyens JP, Bauer JM, Boirie Y, Cederholm T, Landi F, Martin FC, Michel JP, Rolland Y, Schneider SM, et al. Sarcopenia: European consensus on definition and diagnosis: Report of the European Working Group on Sarcopenia in Older People. Age Ageing. 2010;39(4):412-23.

5. Bianco A, Jemni M, Thomas E, Patti A, Paoli A, Ramos Roque J, Palma A Mammina C, Tabacchi G. A systematic review to determine reliability and usefulness of the field-based test batteries for the assessment of physical fitness in adolescents-The ASSO Project. Int J Occup Med Environ Health. 2015;28(3):445-78.

6. Wind AE, Takken T, Helders PJ, Engelbert RH. Is grip strength a predictor for total muscle strength in healthy children, adolescents, and young adults? Eur J Pediatr. 2010;169(3):281-7.

7. Peterson MD, Gordon PM, Smeding S, Visich P. Grip strength is associated with longitudinal health maintenance and improvement in adolescents. J Pediatr. 2018:202:226-30.

8. Fredriksen PM, Mamen A, Hjelle OP, Lindberg M. Handgrip strength in 6-12-year-old children: The Health Oriented Pedagogical Project (HOPP). Scand J Public Health. 2018;46(21_Suppl):54-60.

9. Garcia-Hermoso A, Ramirez-Campillo R, Izquierdo M. Is muscular fitness associated with future health benefits in children and adolescents? A systematic review and meta-analysis of longitudinal studies. Sports Med. 2019;49(7):1079-94

10. Li S, Zhang R, Pan G, Zheng L, Li C. Handgrip strength is associated with insulin resistance and glucose metabolism in adolescents: evidence from National Health and Nutrition Examination Survey 2011 to 2014. Pediatr Diabetes. 2018;19(3):375-80.

11. Agostinis-Sobrinho C, Garcia-Hermoso A, Ramirez-Velez R, Moreira C, Lopes L, Oliveira-Santos J, Povoas SC, Mota J, Santos R. Longitudinal association between ideal cardiovascular health status and muscular fitness in adolescents: the LabMed Physical Activity Study. Nutr Metab Cardiovasc Dis. 2018:28(9):892-9.
12. Steffl M, Chrudimsky J, Tufano JJ. Using relative handgrip strength to identify children at risk of sarcopenic obesity. PLOS ONE. 2017:12(5):e0177006

13. Meng $P$, Hu YX, Fan L, Zhang Y, Zhang MX, Sun J, Liu Y, Li M, Yang Y, Wang $\mathrm{LH}$, et al. Sarcopenia and sarcopenic obesity among men aged 80 years and older in Beijing: prevalence and its association with functional performance. Geriatr Gerontol Int. 2014;14(Suppl 1):29-35.

14. Sayer AA, Syddall HE, Dennison EM, Martin HJ, Phillips DI, Cooper C, Byrne $C D$, Hertfordshire C. Grip strength and the metabolic syndrome: findings from the Hertfordshire Cohort Study. QJM. 2007;100(11):707-13.

15. Meng G, Wu H, Fang L, Li C, Yu F, Zhang Q, Liu L, Du H, Shi H, Xia Y, et al. Relationship between grip strength and newly diagnosed nonalcoholic fatty liver disease in a large-scale adult population. Sci Rep. 2016;6:33255.

16. Kang Y, Park S, Kim S, Koh H. Handgrip strength among Korean adolescents with metabolic syndrome in 2014-2015. J Clin Densitom. 2018:23:271-7.

17. Yi DW, Khang AR, Lee HW, Son SM, Kang YH. Relative handgrip strength as a marker of metabolic syndrome: the Korea National Health and Nutrition Examination Survey (KNHANES) VI (2014-2015). Diabetes Metab Syndr Obes. 2018;11:227-40.

18. Kang Y, Park S, Kim S, Koh H. Estimated prevalence of adolescents with nonalcoholic fatty liver disease in Korea. J Korean Med Sci. 2018;33(14):e109.

19. Bohannon RW, Wang YC, Yen SC, Grogan KA. Handgrip strength: a comparison of values obtained from the NHANES and NIH toolbox studies. Am J Occup Ther. 2019;73(2):7302205080p7302205081-7302205080p7302205089.

20. Centers for Disesae Control and Prevention. National Health and Nutrition Examination Survey (NHANES) Muscle Strength Procedures Manual. 2013.

21. Cohen DD, Gomez-Arbelaez D, Camacho PA, Pinzon S, Hormiga C, TrejosSuarez J, Duperly J, Lopez-Jaramillo P. Low muscle strength is associated with metabolic risk factors in Colombian children: the ACFIES study. PLoS ONE. 2014;9(4):e93150.

22. Grontved A, Ried-Larsen M, Moller NC, Kristensen PL, Froberg K, Brage $\mathrm{S}$, Andersen LB. Muscle strength in youth and cardiovascular risk in young adulthood (the European Youth Heart Study). Br J Sports Med. 2015:49(2):90-4.

23. Lee K. Relative handgrip strength in relation to depressive mood and suicidal ideation in Koreans using the 2015 KNHANES data. J Musculoskelet Neuronal Interact. 2018;18(3):333-8.

24. Ramirez-Velez R, Pena-Ibagon JC, Martinez-Torres J, Tordecilla-Sanders A, Correa-Bautista JE, Lobelo F, Garcia-Hermoso A. Handgrip strength cutoff for cardiometabolic risk index among Colombian children and adolescents: the FUPRECOL Study. Sci Rep. 2017;7:42622.

25. Ng AK, Hairi NN, Jalaludin MY, Majid HA. Dietary intake, physical activity and muscle strength among adolescents: the Malaysian Health and Adolescents Longitudinal Research Team (MyHeART) study. BMJ Open. 2019;9(6):e026275.

\section{Publisher's Note}

Springer Nature remains neutral with regard to jurisdictional claims in published maps and institutional affiliations.

Ready to submit your research? Choose BMC and benefit from:

- fast, convenient online submission

- thorough peer review by experienced researchers in your field

- rapid publication on acceptance

- support for research data, including large and complex data types

- gold Open Access which fosters wider collaboration and increased citations

- maximum visibility for your research: over 100M website views per year

At $\mathrm{BMC}$, research is always in progress.

Learn more biomedcentral.com/submissions 